\title{
Factors associated with bacteremia due to multidrug-resistant Gram-negative bacilli in hematopoietic stem cell transplant recipients
}

\author{
M. Garnica, A. Maiolino and M. Nucci \\ Serviço de Hematologia, Hospital Universitário, Universidade Federal do Rio de Janeiro, Rio de Janeiro, \\ RJ, Brasil \\ Correspondence to: M. Nucci, Hospital Universitário Clementino Fraga Filho, UFRJ, Rua Professor \\ Rodolpho Paulo Rocco, 255, 21941-913 Rio de Janeiro, RJ, Brasil \\ Fax: +55-21-2562-2460. E-mail: mnucci@hucff.ufrj.br
}

\begin{abstract}
The epidemiology of bacteremia developing during neutropenia has changed in the past decade, with the re-emergence of Gramnegative (GN) bacteria and the development of multidrug resistance (MDR) among GN bacteria. We conducted a case-control study in order to identify factors associated with bacteremia due to multidrug-resistant Gram-negative (MDRGN) isolates in hematopoietic stem cell transplant recipients. Ten patients with MDRGN bacteremia were compared with 44 patients with GN bacteremia without MDR. Bacteremia due to Burkholderia or Stenotrophomonas sp was excluded from analysis (3 cases), because the possibility of intrinsical resistance. Infection due to MDRGN bacteria occurred in $2.9 \%$ of 342 hematopoietic stem cell transplant recipients. Klebsiella pneumoniae was the most frequent MDRGN (4 isolates), followed by Pseudomonas aeruginosa (3 isolates). Among nonMDRGN, P. aeruginosa was the most frequent agent (34\%), followed by Escherichia coli(30\%). The development of GN bacteremia during the empirical treatment of febrile neutropenia (breakthrough bacteremia) was associated with $M D R(P<0.001$, odds ratio $=$ $32,95 \%$ confidence interval $=5-190$ ) by multivariate analysis. Bacteremia due to MDRGN bacteria was associated with a higher death rate by univariate analysis $(40 \mathrm{vs} 9 \% ; \mathrm{P}=0.03)$. We were unable to identify risk factors on admission or at the time of the first fever, but the occurrence of breakthrough bacteremia was strongly associated with MDRGN bacteria. An immediate change in the antibiotic regimen in such circumstances may improve the prognosis of these patients.
\end{abstract}

Key words: Hematopoietic stem cell transplant; Neutropenia; Gram-negative bacteria; Antibiotic resistance; Risk factors

Research supported by Pró Vita, Rio de Janeiro (M. Garnica), CNPq to M. Nucci (\#300235/93-3), and to A. Maiolino (\#301455/ 2006-6).

Received June 14, 2008. Accepted January 5, 2009

\section{Introduction}

The development of antimicrobial resistance by Gramnegative bacilli is a worldwide phenomenon that has been observed among immunocompetent and immunocompromised hosts, including patients with hematologic malignancies and hematopoietic stem cell transplantation (HSCT) recipients (1). A relationship between resistance and poor outcome has been documented in several settings, and has been attributed mostly to the use of inappropriate antibiotic regimens, or to a delay in starting appropriate treatment (2). This problem is of great concern among HSCT recipients, especially during the early post-trans- plant period, when the presence of severe neutropenia and mucositis predisposes these patients to infection. In this setting, a single-day delay in starting appropriate antibiotics may be associated with high mortality rates $(3,4)$. The identification of risk factors associated with the development of specific types of infection may be an important step to predict infection and guide the implementation of strategies to decrease the incidence and the negative impact of these infections (5). The objective of the present study was to identify factors associated with bacteremia due to multidrug-resistant Gram-negative (MDRGN) bacteria occurring in the early neutropenic post-transplant period of HSCT. 


\section{Patients and Methods}

This was a retrospective study performed at Hospital Universitário Clementino Fraga Filho between July 1994 and January 2005. We performed a case-control study in which cases were those who had developed bacteremia due to at least one MDRGN isolate (see definition below), occurring during the neutropenic phase of HSCT, and controls were those who developed bacteremia due to one or more Gram-negative isolates that were not multidrug-resistant (non-MDRGN), occurring during the same period post-HSCT. All patients were hospitalized and were implanted with a central venous catheter. Cases and controls were compared regarding demographic characteristics (age, gender), underlying disease and its status (active or in clinical remission), co-morbid conditions, type of HSCT (autologous, allogeneic), stem cell source (bone marrow or peripheral blood), conditioning regimen (myeloablative or of reduced intensity), number of admissions in the preceding year, previous infection caused by MDRGN bacteria, transfer from another institution, stay in the intensive care unit, type and duration of central venous catheter, presence, severity and duration of mucositis (World Health Organization criteria) (6), use of parenteral nutrition, granulocyte colony-stimulating factor (G-CSF) or corticosteroids, use of antibiotics in the hospital in the preceding 14 days, and duration of neutropenia $(<500$ neutrophils $/ \mathrm{mm}^{3}$ ). We also analyzed the following outcome variables: clinical complications [shock (arterial hypotension requiring vasoactive drugs), renal failure (requirement of dialysis), respiratory failure (requirement of mechanical ventilation), and sinusoidal obstructive syndrome (hepatomegaly, jaundice and ascites or weigh gain)], duration of fever, antibiotic therapy and hospitalization, and death until day 60 of HSCT. We defined an empirical antibiotic regimen as inadequate if any pathogen isolated during the febrile episode was resistant to the antibiotics. The febrile episodes were classified as fever of unknown origin, bacteremia, microbiologically documented infections without bacteremia, or clinically documented infections (7). Poly-microbial bacteremia was defined if more than one pathogen was isolated from one or more blood cultures during the febrile episode. Breakthrough bacteremia was defined as a bacteremia occurring after at least 1 day of antibiotic therapy. An MDRGN isolate was defined as an isolate that exhibited resistance to at least 2 of the following antibiotics: ceftazidime, cefepime, piperacillin-tazobactam, or a carbapenem (imipenem or meropenem) (8). We chose these antibiotics because they are frequently used in the empirical treatment of febrile neutropenia. In the case of poly-microbial bacteremia caused by an MDRGN and a non-MDRGN isolate, the episode was defined as a case (isolation of MDRGN bacteria). We ex- cluded from analysis episodes of infection caused by Gramnegative bacilli that are possibly intrinsically resistant to these classes of antibiotics (9).

Blood cultures were simultaneously collected by peripheral puncture and by the catheter, and processed with the BacT/ALERT system (Organon Teknika, USA). Antimicrobial resistance was determined using the Vitek automated system (Bio-Merieux, Inc., France) and confirmed by the Kirby-Bauer disk-diffusion method (10). The E-test double strip for extended-spectrum betalactamase (ESBL) has been performed since 2002 for Enterobacteriaceae isolates.

All statistical analyses were performed using the SPSS for Windows software (version 11.0.1, SPSS, Inc., USA). Categorical variables were analyzed by the Fisher exact test or the chi-square test as appropriate, and continuous variables were analyzed by the Mann-Whitney test. Variables with $\mathrm{P}<0.1$ in univariate analysis were entered in a logistic regression model for multivariate analysis using a forward and backward process. All tests were 2-tailed, and a $P$ value of $<0.05$ was considered to be statistically significant.

\section{Results}

During the study period, a total of 342 HSCT were performed, and in 335 (98\%) the patients developed fever during neutropenia. The classification of the febrile episodes was as follows: fever of unknown origin in 147 episodes (44\%), clinically documented in 47 (14\%), microbiologically documented without bacteremia in $18(5 \%)$, and bacteremia in 123 (37\%). Gram-negative bacteremia was diagnosed in 56 episodes ( $45 \%$ of bacteremias), 10 of which were caused by MDRGN isolates ( $2.9 \%$ of all HSCT, $18 \%$ of episodes with Gram-negative bacteremia). There were two bacteremias due to Burkholderia cepacia and one episode due to Stenotrophomas maltophilia. Those episodes were excluded from our analysis as described above. The first MDRGN bacteria was isolated in 1998. The distribution of cases during the study period did not suggest the occurrence of outbreaks. Six of the 10 cases occurred after 2002, and 4 cases were clustered in a one-year period (from June 2003 to June 2004), but were caused by different bacteria.

Eight of the 10 cases were caused by a single MDRGN isolate, and in 2 cases two isolates were recovered, of a total of 12 MDRGN isolates. Klebsiella pneumoniae was the most frequent (4 isolates), followed by Pseudomonas aeruginosa (3 isolates), Citrobacter freudii (2 isolates), and Acinetobacterspp, Enterobacterspp and Escherichia coli (1 isolate each). All isolates were resistant to ceftazidime and cefepime. Resistance to imipenem was observed in 3 isolates (25\%). Susceptibility to piperacillin-tazobactam was assessed in 8 isolates, and all were resistant. Tests to characterize the 
type of resistance, such as presence of AmpC or metallobeta-lactamase, were not performed in most isolates. The Etest double strip for ESBL was performed in four of the eight Enterobacteriaceae isolates, and was positive in three. The other 4 isolates occurred before the implementation of the Etest for ESBL. Resistance to ciprofloxacin and aminoglycosides was observed in 58 and $50 \%$ of the isolates, respectively.
The 10 cases with MDRGN isolates were compared with 44 controls. A total of 44 Gram-negative bacterial isolates were recovered from the 44 controls. $P$. aeruginosa was the most frequent agent (15 isolates, $34 \%$ ), followed by $E$. coli (13 isolates, $30 \%)$, K. pneumoniae (7 isolates, 16\%), Enterobacter sp and Acinetobacter sp (4 each, 9\%) and Morganella morganii (1 isolate, 2\%). As shown in Table 1, factors associated with bacteremia due

Table 1. Univariate analysis of factors associated with multidrug-resistant Gram-negative bacteremia in a case-control study.

\begin{tabular}{|c|c|c|c|}
\hline & Cases $(N=10)$ & Controls $(\mathrm{N}=44)$ & Odds ratio $(95 \% \mathrm{Cl})$ \\
\hline Age & $52(11-63)$ & $43.5(20-66)$ & NA \\
\hline Gender (male:female) & 4:6 & 25:19 & $0.51(0.12-2.05)$ \\
\hline \multicolumn{4}{|l|}{ Underlying disease } \\
\hline Acute myeloid leukemia & $1(10 \%)$ & $1(2 \%)$ & $4.78(0.27-83.7)$ \\
\hline Chronic myeloid leukemia & $1(10 \%)$ & $5(11 \%)$ & $0.87(0.08-8.36)$ \\
\hline Multiple myeloma & $5(50 \%)$ & $17(39 \%)$ & $1.59(0.39-6.31)$ \\
\hline Hodgkin's disease & $3(30 \%)$ & $10(23 \%)$ & $1.46(0.32-6.70)$ \\
\hline Non-Hodgkin's lymphoma & 0 & $11(25 \%)$ & NA \\
\hline \multicolumn{4}{|l|}{ Type of HSCT } \\
\hline Autologous & $9(90 \%)$ & $37(84 \%)$ & $1.78(0.18-15.70)$ \\
\hline Allogeneic, myeloablative & $1(10 \%)$ & $6(14 \%)$ & $0.70(0.07-6.60)$ \\
\hline Allogeneic, reduced intensity & 0 & $1(2 \%)$ & NA \\
\hline \multicolumn{4}{|l|}{ Stem cell source } \\
\hline Bone marrow & $1(10 \%)$ & $7(16 \%)$ & $0.59(0.06-5.40)$ \\
\hline Peripheral blood & $9(90 \%)$ & 37 (84\%) & $1.78(0.18-15.70)$ \\
\hline \multicolumn{4}{|l|}{ Disease status } \\
\hline Active & $5(50 \%)$ & $34(77 \%)$ & $0.29(0.07-1.22)$ \\
\hline In clinical remission & $5(50 \%)$ & $10(23 \%)$ & $3.40(0.82-14.20)$ \\
\hline No. of CD34 (x 106/kg) cells infused & $2.53(1.13-15.21)$ & $3.75(1.06-11.16)$ & NA \\
\hline \multicolumn{4}{|l|}{ Co-morbidities } \\
\hline Diabetes mellitus & $2(20 \%)$ & $1(2 \%)$ & $10.80(0.87-133)$ \\
\hline Chronic renal failure & 0 & $2(4 \%)$ & NA \\
\hline No co-morbidity & $8(80 \%)$ & $41(93 \%)$ & $0.29(0.03-2.04)$ \\
\hline Previous colonization by MDR Gram-negative isolate & 0 & $2(4 \%)$ & NA \\
\hline Duration of G-CSF use (days) & $7.5(0-23)$ & $9(0-18)$ & NA \\
\hline Previous admission in the ICU & $1(10 \%)$ & 0 & NA \\
\hline Karnofsky performance status & $60(30-70)$ & $70(10-100)$ & NA \\
\hline Use of corticosteroids & $5(50 \%)$ & $13(29 \%)$ & $2.38(0.59-9.66)$ \\
\hline Use of total parenteral nutrition & $3(30 \%)$ & $4(9 \%)$ & $4.29(0.78-23.40)$ \\
\hline Previous use of antibiotics & $7(70 \%)^{\star}$ & $5(11 \%)$ & $18.20(3.52-94.00)$ \\
\hline Carbapenem & $1(10 \%)$ & 0 & NA \\
\hline 3rd-generation cephalosporin & $3(30 \%)^{*}$ & 0 & NA \\
\hline 4th-generation cephalosporin & $4(40 \%)^{*}$ & $3(7 \%)$ & $9.11(1.62-51.10)$ \\
\hline Quinolone & $2(20 \%)$ & $3(7 \%)$ & $3.42(0.49-23.80)$ \\
\hline Vancomycin & $3(30 \%)$ & $4(9.1 \%)$ & $4.29(0.78-23.40)$ \\
\hline Metronidazole & $3(30 \%)^{*}$ & $1(2.3 \%)$ & $18.40(1.67-203)$ \\
\hline Aminoglycoside & $2(20 \%)^{*}$ & 0 & NA \\
\hline No. of antibiotics administered & $2(0-9)^{*}$ & $0(0-3)$ & NA \\
\hline No. of days of antibiotics & $7(0-31)^{*}$ & $0(0-18)$ & NA \\
\hline No. of days of mucositis & $3(0-16)$ & $1(0-13)$ & NA \\
\hline Tunneled central venous catheter & $10(100 \%)$ & $35(80 \%)$ & $3.1(0.3-70)$ \\
\hline No. of days of central venous catheter & $33.5(12-97)^{\star}$ & $11(5-213)$ & NA \\
\hline No. of days of neutropenia & $5(0-23)$ & $2(0-13)$ & NA \\
\hline No. of days of hospitalization before bacteremia & $21.5(5-46)^{*}$ & $12(4-26)$ & NA \\
\hline
\end{tabular}

Data are reported as $\mathrm{N}(\%)$ or median (range) as indicated. $\mathrm{NA}=$ not applicable; HSCT = hematopoietic stem cell transplant; MDR = multidrug resistant; G-CSF = granulocyte colony-stimulating factor; ICU = intensive care unit. *P $<0.05$ (Mann-Whitney U-test, chisquare test or Fisher exact test). 
to MDRGN isolates by univariate analysis were: previous use of any antibiotic in the past 14 days $(P<0.001)$, previous use of a 3rd- or 4th-generation cephalosporin $(P$ $=0.005$ and 0.02 , respectively), metronidazole $(P=0.017)$ or aminoglycosides $(P=0.03)$, number of antibiotics received $(P<0.001)$, duration of antibiotic use $(P<0.001)$, longer duration of indwelling venous catheter use $(P=$ $0.001)$, longer duration of hospitalization $(P=0.02)$, and breakthrough bacteremia $(P<0.001)$. Inadequate treatment was observed in 9 cases, and the median time to adequate therapy was 3.5 days. All controls received adequate empirical treatment. Multivariate analysis revealed breakthrough bacteremia as the only variable associated with MDRGN bacteremia $(P<0.001$, OR $=31.9$, $95 \% \mathrm{Cl}=5.32-190)$. This analysis also included duration of hospitalization, duration of central venous catheter, duration and number of antibiotics used, previous use of 3rd- or 4th-generation cephalosporin, metronidazole and aminoglycosides.

Compared to patients with bacteremia due to nonMDRGN isolates, patients with MDRGN bacteremia were more likely to have a clinical complication (50 vs $14 \%, P=$ 0.02). In addition, the death rate of patients with MDRGN bacteremia was higher (40 vs $9 \%, P=0.03$ ). However, a multivariate analysis of risk factors for death (that included gender, age, underlying disease and its status, Karnofsky performance status, co-morbidities, duration of mucositis and neutropenia, adequate empiric antibiotic regimen, invasive fungal infection, and the presence of complications) revealed that an invasive fungal infection $(P=0.04, O R=$ $24.90,95 \% \mathrm{Cl}=1.12-552)$ and shock $(\mathrm{P}=0.02$, OR $=$ $10.60,95 \% \mathrm{Cl}=1.35-82.70$ ) were associated with death, whereas bacteremia due to MDRGN bacteria was marginally significant $(P=0.06, O R=6.70,95 \% C l=0.90-50.60)$.

\section{Discussion}

Our study failed to identify any particular risk factor for MDRGN bacteria related to the type of transplant, underlying disease, or a specific clinical manifestation of infection. The only variable associated with MDRGN bacteremia was breakthrough bacteremia, that is, the occurrence of bacteremia after the start of empirical antibiotic therapy. Patients with breakthrough bacteremia were $>30$ times more likely to have infection due to an MDRGN isolate.

In another study performed to identify risk factors for $P$. aeruginosa MDR in cancer patients, no risk factor was identified when cases were matched with controls for underling disease and date of admission. Since the authors did not analyze the time of positive bacteremia, breakthrough bacteremia was not assessed in their study (11). In another study, Krcméry Jr. et al. (12) assessed risk factors for MDR in neutropenic patients. They found a relationship between previous use of antibiotics (especially imipenem) and infection due to MDRGN bacteria, but the comparison was made with non-MDR infections, and only univariate analysis was performed. Interestingly, they observed more breakthrough bacteremias and inadequate empirical antibiotic therapy among cases (both statistically significant). Another study by the same investigators analyzed $P$. aeruginosa MDR in cancer patients and found acute leukemia, prolonged neutropenia, previous exposure to amikacin, 3rd-generation cephalosporins, imipenem and quinolones as risk factors, but again only univariate analysis was performed (13).

Our study was able to identify only a characteristic of MDRGN infection rather than a specific risk factor. The association between breakthrough bacteremia and MDRGN bacteria may have occurred because the empirical antibiotic regimen was not appropriate to treat or to prevent an infection due to a resistant organism, and not because this antibiotic treatment induced the development of resistance. The occurrence of bacteremia due to MDRGN bacteria would be a reflex of the epidemiological characteristics of the hospital rather than being associated with a specific characteristic of HSCT.

In the present study, MDRGN bacteria were associated with higher mortality by univariate analysis. We did not perform multivariate analysis of risk factors for death because of the small sample size. The most likely explanation of an increased mortality in patients with infection due to MDRGN isolates is that these patients receive inappropriate antibiotic coverage (14-17).

The main limitation of our study is the small sample size. While we could not identify risk factors that could guide in the selection of a different empirical antibiotic regimen in the first fever, our data suggest that once a patient develops a positive blood culture for a Gramnegative isolate during antibiotic therapy, it is very likely that the isolate is MDR. In this setting a change in the regimen is recommended. 


\section{References}

1. Picazo JJ. Management of the febrile neutropenic patient: a consensus conference. Clin Infect Dis 2004; 39 (Suppl 1): S1-S6.

2. Cosgrove SE. The relationship between antimicrobial resistance and patient outcomes: mortality, length of hospital stay, and health care costs. Clin Infect Dis 2006; 42 (Suppl 2): S82-S89.

3. Ramphal R. Changes in the etiology of bacteremia in febrile neutropenic patients and the susceptibilities of the currently isolated pathogens. Clin Infect Dis 2004; 39 (Suppl 1): S25S31.

4. Collin BA, Leather HL, Wingard JR, Ramphal R. Evolution, incidence, and susceptibility of bacterial bloodstream isolates from 519 bone marrow transplant patients. Clin Infect Dis 2001; 33: 947-953.

5. Cordonnier C, Herbrecht R, Buzyn A, Leverger G, Leclercq $R$, Nitenberg $G$, et al. Risk factors for Gram-negative bacterial infections in febrile neutropenia. Haematologica 2005; 90: 1102-1109.

6. Lark RL, McNeil SA, VanderHyde K, Noorani Z, Uberti J, Chenoweth C. Risk factors for anaerobic bloodstream infections in bone marrow transplant recipients. Clin Infect Dis 2001; 33: 338-343.

7. From the Immunocompromised Host Society. The design, analysis, and reporting of clinical trials on the empirical antibiotic management of the neutropenic patient. Report of a consensus panel. J Infect Dis 1990; 161: 397-401.

8. Fluit AC, Schmitz FJ, Verhoef J. Multi-resistance to antimicrobial agents for the ten most frequently isolated bacterial pathogens. Int J Antimicrob Agents 2001; 18: 147-160.

9. Labarca JA, Leber AL, Kern VL, Territo MC, Brankovic LE, Bruckner DA, et al. Outbreak of Stenotrophomonas malto- philia bacteremia in allogenic bone marrow transplant patients: role of severe neutropenia and mucositis. Clin Infect Dis 2000; 30: 195-197.

10. National Committee for Clinical Laboratory Standards. Methods for dilution antimicrobial susceptibility tests for bacteria that grow aerobically. 5th edn. 2006.

11. Ohmagari N, Hanna H, Graviss L, Hackett B, Perego C, Gonzalez V, et al. Risk factors for infections with multidrugresistant $P$ seudomonas aeruginosa in patients with cancer. Cancer 2005; 104: 205-212.

12. Krcméry V Jr, Spanik S, Krupova I, Trupl J, Kunova A, Smid $M$, et al. Bacteremia due to multiresistant Gram-negative bacilli in neutropenic cancer patients: a case controlled study. J Chemother 1998; 10: 320-325.

13. Krcmery V Jr, Trupl J, Kunova A, Spanik S, llavska I, Hel' pianska $\mathrm{L}$, et al. Imipenem-resistant $P$. aeruginosa bacteraemia in cancer patients: risk factors, clinical features and outcome. Bratisl Lek Listy 1996; 97: 647-651.

14. Howard DH, Scott RD, Packard R, Jones D. The global impact of drug resistance. Clin Infect Dis 2003; 36: S4-S10.

15. Howard DH, Scott RD. The economic burden of drug resistance. Clin Infect Dis 2005; 41 (Suppl 4): S283-S286.

16. Hyle EP, Lipworth AD, Zaoutis TE, Nachamkin I, Bilker WB, Lautenbach $\mathrm{E}$. Impact of inadequate initial antimicrobial therapy on mortality in infections due to extended-spectrum beta-lactamase-producing enterobacteriaceae: variability by site of infection. Arch Intern Med 2005; 165: 1375-1380.

17. Kang Cl, Kim SH, Park WB, Lee KD, Kim HB, Kim EC, et al. Bloodstream infections caused by antibiotic-resistant Gramnegative bacilli: risk factors for mortality and impact of inappropriate initial antimicrobial therapy on outcome. Antimicrob Agents Chemother 2005; 49: 760-766. 